\title{
Téoros
}

Revue de recherche en tourisme

\section{Entretien avec Emmanuel Reynard, professeur à l'Université de Lausanne, Institut de géographie de Lausanne}

\section{Mélanie Duval et Christophe Gauchon}

Volume 29, numéro 2, 2010

URI : https://id.erudit.org/iderudit/1024885ar

DOI : https://doi.org/10.7202/1024885ar

Aller au sommaire du numéro

Éditeur(s)

Université du Québec à Montréal

ISSN

0712-8657 (imprimé)

1923-2705 (numérique)

Découvrir la revue

Citer ce document

Duval, M. \& Gauchon, C. (2010). Entretien avec Emmanuel Reynard, professeur à l'Université de Lausanne, Institut de géographie de Lausanne. Téoros, 29(2),

158-159. https://doi.org/10.7202/1024885ar d'utilisation que vous pouvez consulter en ligne. 


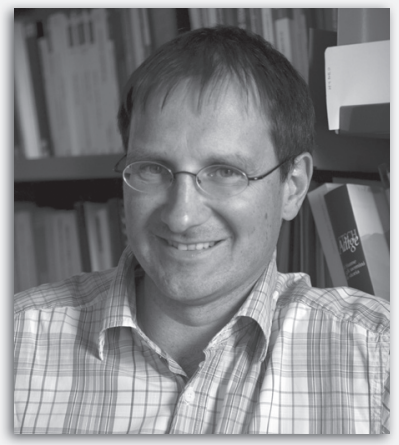

\title{
Entretien \\ avec Emmanuel REYNARD, professeur à l'Université de Lausanne, Institut de géographie de Lausanne
}

\author{
Propos recueillis par Mélanie DUVAL et Christophe GAUCHON
}

\begin{abstract}
Comment avez-vous commencé à travailler sur le géotourisme?
À la base, je ne travaillais pas sur le géotourisme. J'ai d'abord une formation de géomorphologue et j'ai commencé à m'intéresser à ces questions de géotourisme suite à mon travail de licence en 1992. Celui-ci portait sur le site de Tsanfleuron, en Valais, en Suisse. J'avais fait une étude de géomorphologie classique, avec cartographie et reconstitution des stades glaciaires, et j'ai rencontré des glaciologues de l'Université libre de Bruxelles. Ceux-ci travaillaient sur les échanges chimiques entre la glace et le substratum rocheux. De cette rencontre a émergé l'idée de réaliser une brochure didactique qui présenterait le karst et le retrait glaciaire dans cette région : en 1995, on a publié une première édition de cette brochure.

Dans les 10 années qui ont suivi, cette brochure a été diffusée. Lorsqu'elle a été épuisée, on a proposé un concept un peu plus général : il s'agissait de mettre en place des panneaux didactiques traitant de différentes thématiques en plusieurs lieux. On a également réalisé un document à destination des écoles. II est prévu de développer d'autres types de médias comme une carte géotouristique qui devrait être publiée l'été prochain.
\end{abstract}

\section{Aujourd'hui, quels sont les autres projets dans lesquels vous êtes impliqué?}

Un projet sur des thématiques proches porte sur la région de Verbier (Valais). Depuis plusieurs années, on a conduit de nombreux travaux sur le domaine périglaciaire, et on a acquis beaucoup d'expériences et de données. Dans ce cas, il s'agit de mettre l'accent sur les dimensions géomorphologiques du domaine skiable, en partenariat avec la société de remontées mécaniques. Un autre projet, qui est en phase de démarrage, accompagne le projet de parc naturel régional dans le val d'Hérens, au sud de Sion. Là, on a signé une convention avec une des communes et une fondation pour la Maison de la montagne qui vise à créer un centre scientifique montagnard. Pour l'instant, c'est surtout un travail scientifique avec des recherches pour mieux comprendre la géomorphologie de cette région, qui était relativement peu étudiée. Ensuite, il y aura une seconde phase plus axée sur la valorisation.

\section{Avant d'aller plus loin dans cet entretien, quelle est votre définition du géotourisme?}

J'ai une définition assez stricte du géotourisme. Pour moi, c'est une forme de tourisme qui vise à valoriser le patrimoine géologique d'une région. Le géotourisme, comme n'importe quelle forme de tourisme, se base sur une offre primaire de qualité, des paysages aux caractéristiques géologiques de qualité. À mon avis, on ne peut pas faire du géotourisme partout : I'Islande s'y prête mieux qu'une région complètement recouverte de forêt où l'on ne distingue rien ou qu'une région où la géologie est très peu diversifiée. Ensuite, il faut que des biens et des services soient développés pour cette valorisation. Le géodiscours est essentiel. Pour moi, la visite d'un parc national où l'on ne fait que regarder le paysage est une forme de tourisme contemplatif. Si c'est avec des moyens de locomotion durable, ce sera du tourisme doux. Cependant, pour qu'il y ait géotourisme, il faut que la dimension géosciences soit mise en avant. II faut qu'il y ait cet aspect «connaissance du fonctionnement de la Terre » et pas simplement une démarche contemplative.

\begin{abstract}
Suivant cette définition, quel serait aujourd'hui, pour vous, le lieu le plus remarquable concernant le géotourisme?

Parmi les lieux que je connais, je pense au géoparc de l'île de Lesbos, en Grèce. D’abord, il présente un intérêt géologique important parce que c'est une forêt qui a été pétrifiée lors d'une éruption volcanique. Là-dessus, on a greffé toute une série de réalisations de valorisation géotouristique sur les sites eux-mêmes, mais également un centre d'interprétation qui sert de musée et de laboratoire, et qui permet d'organiser des activités avec les scolaires.

Autour de ce site, des dynamiques se sont mises en place : cela a d'abord été un site protégé; il est devenu un géoparc européen; puis il a évolué en un géoparc UNESCO. Autour de ces infrastructures, il y a toute une activité sociale avec de l'artisanat, des produits agricoles ou de la pêche, etc. dans une région qui était plutôt délaissée d'un point de vue économique et démographique. C'est un des cas où on voit les impacts socio-économiques importants que peut avoir le développement géotouristique, avec plusieurs milliers de visiteurs pour un village de 300 habitants. Ce développement géotouristique, qui n'était pas évident, a été porté par des universitaires et a vraiment amené une plus-value locale.
\end{abstract}

En matière de géotourisme, deux tendances semblent s'observer et parfois s'opposer : d'un côté, on assiste à une certaine prolifération des sentiers et des supports pédagogiques, et d'un autre côté, on note une certaine frilosité de la part des acteurs du tourisme à investir ce secteur touristique. Comment peut-on, à votre avis, expliquer cette situation?

À une échelle locale, le géotourisme est lié à une volonté de promotion territoriale. Les collectivités locales se considèrent comme étant en charge de la promotion de leur commune. Sur ce point, je pense qu'Internet a été un moteur dans le changement de perception de l'activité que doit avoir une administration publique qui, au niveau local, se sent en charge de créer, de gérer une image. Ce n'est pas quelque chose de nouveau, mais c'est une tendance qui s'est accélérée ces dernières années. Maintenant, n'importe quel maire, tout comme l'envie d'ouvrir une route ou d'inaugurer un pont, veut faire une réalisation didactique qui présente les qualités de sa commune; cela fait partie de ses missions. Ce phénomène n'est pas limité au géotourisme, mais il a été un moyen d'impulser des réalisations géotouristiques.

Dans le même temps, les contacts avec les professionnels du tourisme restent peu nombreux, et j'y vois essentiellement deux raisons. D'abord, le géotourisme est parti des géosciences : à un moment, cela a été perçu comme un moyen de renouveler la recherche. Ce sont les géosciences qui ont créé l'offre alors qu'il n'y avait pas vraiment une réelle demande provenant des milieux touristiques. Ensuite, il faut y voir aussi certainement une raison liée aux logiques de rentabilité économique. Dans les régions montagneuses, je vois bien cette volonté de moins dépendre du ski, de développer le tourisme d'été, le tourisme doux. Par contre, il y a un tel gouffre en matière de rentrées économiques entre la saison hivernale et le tourisme d'été qu'il n'y a pas encore un véritable engouement pour développer des alternatives. 
Il existe donc un intérêt poli pour le géotourisme, mais pas de réel intérêt économique fort. Une telle analyse n'est toutefois certainement pas valable pour des régions sans tourisme l'hiver.

\section{Du fait de ce décalage et de ce relatif désintérêt des professionnels du tourisme, est-ce que cela ne pose pas des soucis en termes de diffusion et de promotion touristique? Si l'on reprend les actions auxquelles vous avez participé et notamment cette brochure sur Tsanfleuron, comment celle-ci a été diffusée?}

Elle a été très mal diffusée, ce qui est bien malheureux et qui est en partie de ma responsabilité. En tant que géomorphologue, je ne me suis jamais occupé de l'aspect promotion. Et c'est valable pour tous les projets dans lesquels j'ai été impliqué : j'ai fait le travail scientifique, l'élaboration, éventuellement le travail de mise en page des brochures avec les imprimeurs, mais je ne me suis jamais occupé de la partie suivante, de la diffusion, etc.

\section{De ce fait, pour les deux autres projets actuellement en cours,} comment cette question de la diffusion va-t-elle être traitée?

Étant donné les lacunes précédemment identifiées, cette question de la diffusion est un enjeu majeur dans les travaux en cours et à venir. Ainsi, un travail de diplôme de l'Université de Lausanne a été réalisé sur quatre sentiers didactiques dans lesquels I'Institut de géographie de Lausanne avait été d'une manière ou d'une autre impliqué (Berrebi, 2008). Ce travail a permis de retracer l'histoire de ces quatre projets : d'où était venue l'idée de ce projet, comment celui-ci s'est mis en place? L'étudiante a également fait tester les quatre sentiers par des groupes de personnes pour évaluer la qualité des sentiers développés. Et une des conclusions est justement, dans la plupart des cas, que ces aspects de promotion, de diffusion, de maintenance n'étaient pas suffisamment pris en compte.

\section{Quels sont donc d'après vous les principaux enjeux en matière de géotourisme?}

En tout, j'en vois quatre. Le premier est lié à la dimension territoriale du géotourisme et à la question de la plus-value économique. Le géotourisme étant une forme de tourisme, il s'inscrit nécessairement dans une dimension économique. Si l'on veut créer des retombées économiques pour la région concernée, il faut mieux connaitre la part de marché que peut représenter le secteur géotouristique, en fonction de la définition que l'on en a retenue. Si l'on prend l'exemple des sentiers à thème, ce n'est pas forcément du géotourisme. En ce moment, dans ma commune, je m'occupe d'un bisse, un canal d'irrigation qui est revalorisé pour le tourisme, avec des vestiges d'anciens canaux accrochés aux parois. On a développé un sentier dans les parois sur un parcours très aérien qui reçoit environ 4000 visiteurs par semaine durant tout l'été. On n'a pas fait d'études précises sur les retombées économiques, mais elles sont très importantes pour les restaurants, par exemple. Cependant, ce n'est pas de la géologie; on est davantage dans le registre d'un tourisme culturel que dans celui du géotourisme.

Le second enjeu est lié à la question de l'entretien des réalisations. Généralement, dans des projets de réalisations géotouristiques, on définit un budget pour la phase de réalisation, mais peu pour la promotion touristique et quasiment rien n'est prévu pour assurer l'entretien des installations dans le temps. Certains panneaux qui ont été installés il y a 10 ans sont déjà défraîchis; ils n'ont pas résisté aux conditions atmosphériques.

Le troisième enjeu est relatif à la qualité des médias et des réalisations : brochures, panneaux, guidage... À mon sens, on trouve des réalisations de qualités très différentes. Aujourd'hui, dans le canton du Valais, il existe d'ailleurs un projet de certification des sentiers. Dans les 15 dernières années, il y a vraiment eu une prolifération de ces réalisations, et parfois la qualité scientifique n'était pas au rendez-vous. Un mandat a d'ailleurs été donné à mes collègues de l'Université de Lausanne pour développer des indicateurs afin de mettre en place une méthode d'évaluation des réalisations géotouristiques.

Enfin, le dernier enjeu est lié à la prise en compte des attentes du public ou même des publics. Pour l'instant, il y a peu de travaux à ma connaissance qui portent sur cela. Est-ce que les réalisations correspondent aux attentes des publics ciblés? Souvent, elles sont faites sans études préalables des publics. La partie «tourisme» du géotourisme doit être menée de manière plus professionnelle, avec des études de marché, du marketing, et pas seulement le regard du spécialiste en géosciences. Sur ce point, j'ai un regard assez critique sur notre pratique.

\section{Comment prendre en compte les niveaux disparates de connaissances des touristes?}

Je pense que cela passe par une analyse des différents types de public. Quand on fait une réalisation géotouristique, il faut déjà se poser la question des visiteurs auxquels on veut s'adresser, si ce sont des enfants, des familles ou des personnes âgées, par exemple. D'une part, il y a vraiment une nécessité de faire des études sur l'intérêt que suscitent des réalisations existantes auprès des visiteurs. D'autre part, pour des réalisations futures, il y a la nécessité de faire des analyses de marché et de s'interroger sur les publics ciblés.

Et enfin, une troisième voie que je perçois, ce sont les travaux qui portent actuellement sur la médiation, sur les techniques, les méthodes utilisées pour faire cette médiation dans le domaine des sciences de la Terre.

Pour ce faire, travaillez-vous avec des collègues d'autres disciplines? Oui et non, pas suffisamment, je dirais. Pour l'instant, nous avons à Lausanne quatre doctorants qui travaillent sur ces questions de médiation : Amandine Perret travaille sur l'inventaire des ressources géologiques du Chablais, Lenka Kozlik sur la médiation et Simon Martin sur la géovisualisation de la géomorphologie, sur l'utilisation des nouvelles techniques de visualisation comme Google Earth et d'autres nouveaux moyens informatiques. Un des volets de ses recherches porte sur les questions sémiologiques et un second volet passe par des tests auprès des publics potentiels. Géraldine Regolini-Bissig travaille dans le même sens, mais plutôt sur les cartes avec des publics scolaires, mais aussi avec des touristes : comment établissent-ils des liens entre les cartes et les paysages observés? Elle arrive à des résultats vraiment intéressants : certains modes de représentation ne sont pas du tout agréés, car les touristes n'arrivent pas bien à saisir le relief avec des courbes de niveau alors qu'ils le saisissent beaucoup mieux avec des représentations ombrées, introduisant de la perspective. Pour l'instant, ces travaux se déroulent dans le cadre de l'Institut de géographie, et les contacts avec d'autres collègues des sciences sociales ou des sciences du tourisme ne sont pas suffisamment développés. Ce serait une prochaine étape que d'arriver à mettre en place des collaborations pluridisciplinaires. Ce serait vraiment là qu'il y aurait à mon avis une plus-value scientifique intéressante.

\section{Bibliographie des travaux de l'Institut de géographie de Lausanne sur le géotourisme :}

BERREBI, Y. (2008) «Les sentiers didactiques. Analyse de la perception du public face à quatre réalisations géodidactiques". Mémoire de licence en géographie, Lausanne : Université de Lausanne, Institut de géographie (téléchargeable sur <http://mesoscaphe.unil.ch/igul/memoires/bd/>).

MARTIN, Simon et Emmanuel REYNARD (2009) How can a complex geotourist map be made more effective? Popularisation of the Tsanfleuron heritage Nalais, Switzerland): 6th European Congress on Regional Geoscientific Cartography and Information Systems (Munich, 9 au 12 juin 2009), Proceedings, vol. 2, p. 261-264.

REYNARD, Emmanuel (2006) «Valorisation géotouristique du karst de Tsanfleuron », DANS Gestion durable de l'environnement karstique, Actes de la réunion annuelle de la Société suisse de Géomorphologie (La Chaux-de-Fond, 3-4 septembre 2004), R. LUGON (éd.), p. 69-79. Sion : Institut Universitaire Kurt Bösch.

REYNARD, Emmanuel (2008) «Le lapiaz de Tsanfleuron. Un paysage glacio-karstique à protéger et à valoriser », DANS «Karsts de montagne. Géomorphologie, patrimoine et ressources", F. HOBLÉA, Emmanuel REYNARD et J.-J. DELANNOY (éd.), p. 157-168. Chambéry, Coll. «Edytem», Cahiers de Géographie, no 7.

REYNARD, Emmanuel (2008) «Scientific research and tourist promotion of geomorphological heritage», Geografia Fisica e dinamica Quaternaria, vol. 31, p. 225-230. REYNARD, Emmanuel et Y. BERREBI (2008) Percorsi geodidattici e aspettative del pubblico: Geologia e turismo : beni geologici e geodiversità, Atti del Terzo congresso nazionale Geologia e Turismo (Bologne 1-3 mars 2007), p. 15-21. 